TRANSACTIONS OF THE

AMERICAN MATHEMATICAL SOCIETY

Volume 348, Number 8, August 1996

\title{
AN ALGEBRAIC APPROACH TO MULTIPARAMETER SPECTRAL THEORY
}

\author{
LUZIUS GRUNENFELDER AND TOMAŽ KOŠIR
}

\begin{abstract}
Root subspaces for multiprameter eigenvalue problems are described using coalgebraic techniques. An algorithm is given to construct bases for the root subspaces.
\end{abstract}

\section{INTRODUCTION}

Multiparameter spectral theory has its beginnings in the works of Klein and Hilbert, among others, at the turn of the century. They considered boundary value problems of Sturm-Liouville type that yield, after the method of separation of variables is employed, a two-parameter system of partial differential equations that have separated variables but are linked by parameters. One of the problems considered then is to obtain completeness and expansion theorems. A more detailed survey of the developments in multiparameter spectral theory for partial differential equations can be found in [10] (and also in the review [24]). In the 1960s Atkinson [5] introduced an abstract algebraic setting for multiparameter spectral theory that revived the theory. We refer to the monographs [12, 23, 27] and lecture notes [17] for recent developments in multiparameter spectral theory.

In one-parameter spectral theory one shows that Jordan chains span the generalized eigenspaces (also called root subspaces) and that they form a complete basis thus yielding various completeness and expansion theorems. In the multiparameter setting this problem was already stated by Atkinson [4] and considered by Isaev [16] and Gadziev [11]. But it remained, in general, an open problem even in the finite-dimensional case. An important contribution was made by Binding [6] who solved the problem for real eigenvalues of the so-called uniformly elliptic case in a general Hilbert space setting. With Košir [8] they found a basis for the "second" root subspace of multiparameter eigenvalue problems of Fredholm type. Faierman [10] studied root subspaces for two-parameter boundary value problems of the Sturm-Liouville type. In the finite-dimensional setting the problem was solved in $[19,20]$ for root subspaces of simple eigenvalues, i.e., eigenvalues with one-dimensional eigenspace.

The methods used in these papers vary. For example, in [6] analytic perturbation theory is used (similarly in $[7,9]$ where dimensions of root subspaces are studied), while in $[19,20]$, partially also in [8], methods of linear algebra are used.

Received by the editors May 2, 1994.

1991 Mathematics Subject Classification. Primary 16W30, 35P10, 47A13.

(C)1996 American Mathematical Society 
Our main objective in this paper is to describe the root subspaces for multiparameter systems of the form

$$
W_{i}(\boldsymbol{\lambda})=\sum_{j=1}^{n} V_{i j} \lambda_{j}-V_{i 0}(i=1,2, \ldots, n) .
$$

Here $V_{i j}$ are linear transformations on a vector space $H_{i}$ over a field $F$ and $\boldsymbol{\lambda}=$ $\left(\lambda_{1}, \lambda_{2}, \ldots, \lambda_{n}\right)$ are parameters. The usual eigenvalue problem for a single linear transformation $V$ can be considered via the comparison of $V$ to $M_{\lambda}$, the map 'multiplication by $\lambda$ ' on the polynomial algebra $F[\lambda]$. Here we use the dual map $M_{\lambda}^{*}$ in place of $M_{\lambda}$. This idea is used by Grunenfelder and Omladič [14] to develop an algebraic approach to study operator polynomials. (See also [13], where commuting linear transformations are studied.) It is similar to the idea of replacing $\lambda$ by a formal partial derivative $\frac{\partial}{\partial \lambda}$, which was suggested for the multiparameter eigenvalue problems by Atkinson $[4, \S 5]$ and was used by Gadziev [11, 12]. The dual map $M_{\lambda}^{*}$ acts on the vector space $F[\lambda]^{\circ}$ of representative functionals on $F[\lambda]$ and is a derivation with respect to the algebra structure on $F[\lambda]^{\circ}$. However it is the coalgebra structure on $F[\lambda]^{\circ}$ (see $[1,25]$ ) that enables us to prove our main result. We associate with a system (1) a set of comodules. Our main result is then that the root subspaces associated with (1) are described by the cotensor product [15] of the associated comodules. This result generalizes Atkinson's theorem [5] that eigenspaces associated with (1) at $\boldsymbol{\lambda} \in F^{n}$ are given by the tensor product of the kernels of the $W_{i}(\boldsymbol{\lambda})$ 's.

Our paper is organized as follows. First we consider the finite-dimensional case. We introduce Atkinson's approach to multiparameter eigenvalue problems in $\S 2$. Coalgebras and comodules with the properties needed in the sequel are discussed in $\S 3$ and $\S 4$, respectively. In $\S 5$ we present our main result. The structure of elements in the root subspaces for the two-parameter case is studied in $\S 6$. In $\S 7$ we present the algorithm for constructing bases of the root subspaces of two-parameter systems, and we illustrate the algorithm with an example. In the last section the main result is extended to the case of eigenvalues of semi-Fredholm type in the Hilbert space setting. This setting was studied in [8] and arises naturally in various applications, in particular applications to boundary value problems $[3,10]$.

\section{Multiparameter Systems}

Consider a multiparameter system of the form (1), where the linear transformations $V_{i j}(j=0,1, \ldots, n)$ act on a vector space $H_{i}(i=1,2, \ldots, n)$ of finite dimension. The linear transformation $V_{i j}$ on $H_{i}$ induces a linear transformation $V_{i j}^{\dagger}$ on the tensor product $H=H_{1} \otimes H_{2} \otimes \cdots \otimes H_{n}$. It is defined by

$$
V_{i j}^{\dagger}\left(x_{1} \otimes x_{2} \otimes \cdots \otimes x_{n}\right)=x_{1} \otimes \cdots \otimes x_{i-1} \otimes V_{i j} x_{i} \otimes x_{i+1} \otimes \cdots \otimes x_{n}
$$

on a decomposable tensor $x_{1} \otimes x_{2} \otimes \cdots \otimes x_{n} \in H$, where $x_{i} \in H_{i}$, and is extended to $H$ by linearity.

The determinant

$$
\Delta_{0}=\left|\begin{array}{cccc}
V_{11}^{\dagger} & V_{12}^{\dagger} & \cdots & V_{1 n}^{\dagger} \\
V_{21}^{\dagger} & V_{22}^{\dagger} & \cdots & V_{2 n}^{\dagger} \\
\vdots & \vdots & & \vdots \\
V_{n 1}^{\dagger} & V_{n 2}^{\dagger} & \cdots & V_{n n}^{\dagger}
\end{array}\right|
$$


is a linear transformation acting on $H$. It is well defined because any two entries from distinct rows in (3) commute. Similarly we define linear transformations $\Delta_{i}$ $(i=1,2, \ldots, n)$ by replacing the $i$-th column in (3) by $\left[V_{k 0}^{\dagger}\right]_{k=1}^{n}$.

A multiparameter system is called regular if $\Delta_{0}$ is invertible. We assume throughout this paper that this is the case. Note that Atkinson [5] uses the assumption that some linear combination of the $\Delta_{i}(i=0,1, \ldots, n)$ is invertible. But then one can achieve by a shift in parameters $\boldsymbol{\lambda}$ that $\Delta_{0}$ is invertible. We also consider the linear transformation $V: H^{n} \longrightarrow H^{n}$ defined by

$$
V=\left[\begin{array}{cccc}
V_{11}^{\dagger} & V_{12}^{\dagger} & \cdots & V_{1 n}^{\dagger} \\
V_{21}^{\dagger} & V_{22}^{\dagger} & \cdots & V_{2 n}^{\dagger} \\
\vdots & \vdots & & \vdots \\
V_{n 1}^{\dagger} & V_{n 2}^{\dagger} & \cdots & V_{n n}^{\dagger}
\end{array}\right]
$$

and its adjoint transformation

$$
A=\left[\begin{array}{cccc}
\Delta_{011} & \Delta_{012} & \cdots & \Delta_{01 n} \\
\Delta_{021} & \Delta_{022} & \cdots & \Delta_{02 n} \\
\vdots & \vdots & & \vdots \\
\Delta_{0 n 1} & \Delta_{0 n 2} & \cdots & \Delta_{0 n n}
\end{array}\right]
$$

where $\Delta_{0 i j}$ is the $(j, i)$-th cofactor of $\Delta_{0}$. Since the product

$$
A V=\left[\begin{array}{cccc}
\Delta_{0} & 0 & \cdots & 0 \\
0 & \Delta_{0} & \cdots & 0 \\
\vdots & \vdots & & \vdots \\
0 & 0 & \cdots & \Delta_{0}
\end{array}\right]
$$

is invertible, $V$ is invertible.

The linear transformations $\Gamma_{i}=\Delta_{0}^{-1} \Delta_{i}(i=1,2, \ldots, n)$ on $H$ are called the associated transformations of a multiparameter system (1). Here are some of their properties. For the proofs and further properties we refer to [5, Chapter 6].

The linear transformations $\Gamma_{i}$ commute, i.e., $\Gamma_{i} \Gamma_{j}=\Gamma_{j} \Gamma_{i}$ for all $i$ and $j$, and we have

$$
\sum_{j=1}^{n} V_{i j}^{\dagger} \Gamma_{j}=V_{i 0}^{\dagger}
$$

$(i=1,2, \ldots, n)$, or equivalently, the diagram

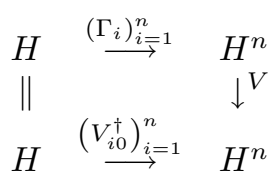

commutes. Note that (5) (and hence (6)) can be viewed as a generalization of Cramer's rule for a system of linear equations.

Next we define the notions of eigenvalues and spectra. An $n$-tuple $\boldsymbol{\lambda} \in F^{n}$ is called an eigenvalue of a multiparameter system (1) if all $W_{i}(\boldsymbol{\lambda})$ are singular. The set of all eigenvalues is called the spectrum of (1). An $n$-tuple $\boldsymbol{\lambda}=\left(\lambda_{1}, \lambda_{2}, \ldots, \lambda_{n}\right) \in$ 
$F^{n}$ is called an eigenvalue of the system of commuting linear transformations $\left\{\Gamma_{i}\right\}$ if

$$
R_{0}=\bigcap_{i=1}^{n} \operatorname{ker}\left(\Gamma_{i}-\lambda_{i} I\right) \neq\{0\}
$$

The set of all these eigenvalues is called the spectrum of the system $\left\{\Gamma_{i}\right\}$. It was proved by Atkinson [5, Theorem 6.9.1] that the spectrum of (1) and that of its associated system of linear transformations $\left\{\Gamma_{i}\right\}$ coincide and that for a given eigenvalue $\boldsymbol{\lambda}$

$$
R_{0}=\operatorname{ker} W_{1}(\boldsymbol{\lambda}) \otimes \operatorname{ker} W_{2}(\boldsymbol{\lambda}) \otimes \cdots \otimes \operatorname{ker} W_{n}(\boldsymbol{\lambda}) .
$$

The subspace $R_{0}$ is called the eigenspace (or the 0-th root subspace) corresponding to $\boldsymbol{\lambda}$. Here we differ from the more usual notation where our $R_{0}$ is considered as the first root subspace (see [8]). The reason for doing so will become clear later. Our main goal in this paper is to describe the higher root subspaces

$$
R_{l}=\bigcap_{m \in M_{l+1}} \operatorname{ker}\left[m\left(\Gamma_{1}-\lambda_{1} I, \Gamma_{2}-\lambda_{2} I, \ldots, \Gamma_{n}-\lambda_{n} I\right)\right],
$$

for $l \geq 1$, in terms of the underlying multiparameter system (1). Here $M_{l}$ is the set of all monomials in $n$ variables of degree $l$. The least integer $l$ such that $R_{l}=R_{l+1}$ is called the ascent of $\boldsymbol{\lambda}$.

To find a description of the subspaces $R_{l}$ in terms of the original transformations $V_{i j}$ has been an open problem of multiparameter spectral theory for some time (cf. [4] and [17, Lecture 6, Problem 4]). We shall use coalgebraic techniques to give such a description. But before we can proceed some notions from the theory of coalgebras and comodules are needed.

\section{Coalgebras}

A coalgebra $\mathcal{C}$ is a vector space with a structure dual to that of an algebra, i.e., with a counit $\varepsilon: \mathcal{C} \rightarrow F$ and a comultiplication $\delta: \mathcal{C} \rightarrow \mathcal{C} \otimes \mathcal{C}$, which are linear maps, such that the diagrams

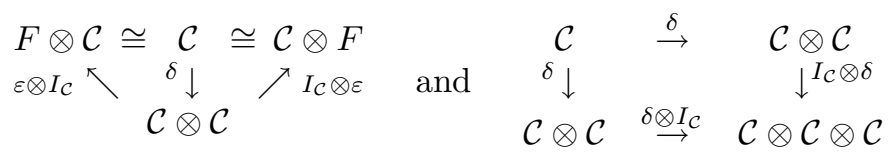

commute. The maps $\varepsilon$ and $\delta$ are called structure maps of $\mathcal{C}$. The first of the above diagrams is the counit law and the second is coassociativity. Here we use the symbol $I_{\mathcal{C}}$ to denote the identity map on $\mathcal{C}$. Note that these diagrams are dual (i.e. the arrows are reversed) to the usual unit law and associativity of algebras. If also the diagram

$$
\begin{array}{rll}
\mathcal{C} & \stackrel{\delta}{\rightarrow} & \mathcal{C} \otimes \mathcal{C} \\
\delta \downarrow & \nearrow \sigma_{12} \\
\mathcal{C} \otimes \mathcal{C} &
\end{array}
$$

commutes we say that $\mathcal{C}$ is cocommutative. Here $\sigma_{i j}$ switches the $i$-th and $j$-th tensor factor. If $\mathcal{C}_{1}$ and $\mathcal{C}_{2}$ are two coalgebras with structure maps $\varepsilon_{1}, \delta_{1}$ and $\varepsilon_{2}, \delta_{2}$, respectively, then $\mathcal{C}_{1} \otimes \mathcal{C}_{2}$ is a coalgebra with structure maps $\varepsilon_{1} \otimes \varepsilon_{2}$ and $\sigma_{23}\left(\delta_{1} \otimes \delta_{2}\right)$.

Suppose that $\mathcal{A}$ is an algebra with unit $\kappa: F \rightarrow \mathcal{A}$ and multiplication $\mu$ : $\mathcal{A} \otimes \mathcal{A} \rightarrow \mathcal{A}$. If $\mathcal{A}$ is finite-dimensional then the dual maps $\kappa^{*}: \mathcal{A}^{*} \rightarrow F$ and 
$\mu^{*}: \mathcal{A}^{*} \rightarrow \mathcal{A}^{*} \otimes \mathcal{A}^{*}$ make $\mathcal{A}^{*}$ into a coalgebra. But if $\mathcal{A}$ is not finite-dimensional then the canonical map $\mathcal{A}^{*} \otimes \mathcal{A}^{*} \rightarrow(\mathcal{A} \otimes \mathcal{A})^{*}$ is not bijective and $\mathcal{A}^{*}$ is not a coalgebra. However, the subspace of representative functionals $\mathcal{A}^{\circ}$ is a coalgebra, called the dual coalgebra of $\mathcal{A}$, with the restrictions of $\kappa^{*}$ and $\mu^{*}$ as structure maps. A functional $f: \mathcal{A} \rightarrow F$ is called representative if its kernel contains an ideal of finite codimension. If $\mathcal{A}$ and $\mathcal{B}$ are two algebras then

$$
(\mathcal{A} \otimes \mathcal{B})^{\circ} \cong \mathcal{A}^{\mathrm{o}} \otimes \mathcal{B}^{\circ}
$$

(see $[1,25])$.

Of particular importance for us are the polynomial algebra $F[x]$ and its dual coalgebra $F[x]^{\circ}$. The vector space $F[x]^{\circ}$ of all representative functionals on $F[x]$ has a (topological) basis $\left\{e_{m}\right\}_{m=0}^{\infty}$, where $e_{m}\left(x^{n}\right)=\delta_{m n}$ and $\delta_{m n}$ is the Kronecker symbol. Every element $f \in F[x]^{\circ}$ has an infinite series representation $f=\sum_{m=0}^{\infty} \alpha_{m} e_{m}$, where $\left\{\alpha_{m}\right\}_{m=0}^{\infty}$ forms a linearly recursive sequence (see [14, 22] for details). The canonical (cocommutative) coalgebra structure on $F[x]^{0}$ is given by

$$
\varepsilon\left(e_{m}\right)=\delta_{0 m} \text { and } \delta\left(e_{m}\right)=\sum_{r+s=m} e_{r} \otimes e_{s}
$$

There is also a multiplication on $F[x]^{\circ}$ defined by

$$
e_{k} * e_{l}=\left(\begin{array}{c}
k+l \\
k
\end{array}\right) e_{k+l} \text {. }
$$

The structure maps (10) and (11) are extended to the whole of $F[x]^{\circ}$ by (infinite) linearity, e.g.

$$
\delta\left(\sum_{m=0}^{\infty} \alpha_{m} e_{m}\right)=\sum_{m=0}^{\infty} \alpha_{m} \delta\left(e_{m}\right) .
$$

Suppose that $\mathcal{I}$ is the ideal of $F[x]$ generated by $(x-\lambda)^{l+1}$. Then $\mathcal{I}$ is of finite codimension in $F[x]$ and the vector space $\mathcal{B}_{\lambda}^{(l)}=(F[x] / \mathcal{I})^{*}$ of all linear functionals on $F[x] / \mathcal{I}$ is a subcoalgebra of $F[x]^{\circ}$. The union

$$
\mathcal{B}_{\lambda}=\bigcup_{l=0}^{\infty} \mathcal{B}_{\lambda}^{(l)}
$$

is an irreducible component of $F[x]^{\circ}$, and if $F$ is algebraically closed then

$$
F[x]^{\mathrm{o}}=\bigoplus_{\lambda \in F} \mathcal{B}_{\lambda}
$$

(see $\left[14\right.$, Theorem 3.2(a)]). The coalgebra $\mathcal{B}_{\lambda}^{(l)}$ has a basis $\left\{e_{i}(\lambda)\right\}_{i=0}^{l}$, where $e_{0}(\lambda)=\sum_{j=0}^{\infty} \lambda^{j} e_{j}$ and $e_{i}(\lambda)=e_{0}(\lambda) * e_{i}(i \geq 1)$. Note that $e_{i}=e_{i}(0)$.

Next define the linear map $D: F[x]^{\circ} \rightarrow F[x]^{\circ}$ as the dual of the map multiplication by $x$ on $F[x]$, that is,

$$
D f(p)=f(x p),
$$

for $p \in F[x]$. This map is a derivation on $F[x]^{\circ}$, i.e., $D(f * g)=D f * g+f * D g$. Since $D e_{i}=e_{i-1}$, we see that

$$
D e_{i}(\lambda)=\lambda e_{i}(\lambda)+e_{i-1}(\lambda) .
$$

Here $e_{-1}(\lambda)=0$. 
The polynomial algebra $F[x]$ and its dual coalgebra of representative functionals have an even richer structure then the one just described. Because it is our intention to introduce only the notions we need later in our discussion we do not develop this theory any further. We refer the interested reader to [14, 22].

By (9) the dual coalgebra of representative functionals on a polynomial algebra $F\left[x_{1}, x_{2}, \ldots, x_{n}\right]$ is

$$
F\left[x_{1}, x_{2}, \ldots, x_{n}\right]^{\mathrm{o}}=F\left[x_{1}\right]^{\mathrm{o}} \otimes F\left[x_{2}\right]^{\mathrm{o}} \otimes \cdots \otimes F\left[x_{n}\right]^{\mathrm{o}} .
$$

Suppose that $\boldsymbol{\lambda}=\left(\lambda_{1}, \lambda_{2}, \ldots, \lambda_{n}\right) \in F^{n}$. Then the tensor product of coalgebras

$$
\mathcal{B}_{\boldsymbol{\lambda}}=\mathcal{B}_{\lambda_{1}} \otimes \mathcal{B}_{\lambda_{2}} \otimes \cdots \otimes \mathcal{B}_{\lambda_{n}}
$$

is a subcoalgebra of $F\left[x_{1}, x_{2}, \ldots, x_{n}\right]^{\mathrm{O}}$, and again

$$
F\left[x_{1}, x_{2}, \ldots, x_{n}\right]^{\circ}=\bigoplus_{\lambda \in F^{n}} \mathcal{B}_{\boldsymbol{\lambda}}
$$

if $F$ is algebraically closed. The filtration (12) induces the tensor product filtration

$$
\mathcal{B}_{\boldsymbol{\lambda}}=\bigcup_{l=0}^{\infty} \mathcal{B}_{\lambda}^{(l)}
$$

where

$$
\mathcal{B}_{\lambda}^{(l)}=\sum_{k_{1}+\cdots+k_{n}=l} \mathcal{B}_{\lambda_{1}}^{\left(k_{1}\right)} \otimes \mathcal{B}_{\lambda_{2}}^{\left(k_{2}\right)} \otimes \cdots \otimes \mathcal{B}_{\lambda_{n}}^{\left(k_{n}\right)}
$$

As in (13) we get derivations $D_{j}: F\left[x_{1}, x_{2}, \ldots, x_{n}\right]^{\mathrm{o}} \rightarrow F\left[x_{1}, x_{2}, \ldots, x_{n}\right]^{\mathrm{o}}$ defined by

$$
D_{j} f(p)=f\left(x_{j} p\right)
$$

for $p \in F\left[x_{1}, x_{2}, \ldots, x_{n}\right]$ and for $j=1,2, \ldots, n$,

\section{Comodules and the Cotensor Product}

A notion dual to the notion of a module over an algebra is the notion of a comodule over a coalgebra. Suppose that $\mathcal{C}$ is a coalgebra. Then a vector space $\mathcal{N}$ is a $\mathcal{C}$-comodule if there is a linear map $\alpha: \mathcal{N} \rightarrow \mathcal{N} \otimes \mathcal{C}$, called a coaction of $\mathcal{C}$ on $\mathcal{N}$, such that the diagrams

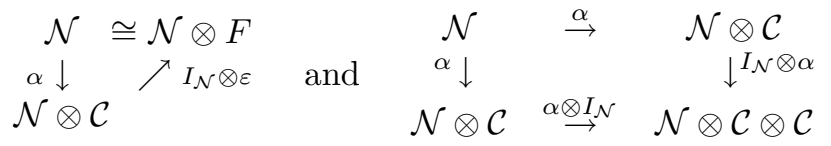

commute. If $H$ is a vector space and $\mathcal{C}$ a coalgebra then the comultiplication $\delta$ of $\mathcal{C}$ induces a coaction $\alpha=I_{H} \otimes \delta$ on $H \otimes \mathcal{C}$. Such a comodule $H \otimes \mathcal{C}$ is called cofree. If $\mathcal{M}$ and $\mathcal{N}$ are $\mathcal{C}$-comodules with coactions $\alpha_{\mathcal{M}}$ and $\alpha_{\mathcal{N}}$, respectively, then a linear $\operatorname{map} \varphi: \mathcal{M} \rightarrow \mathcal{N}$ is called a comodule homomorphism if the diagram

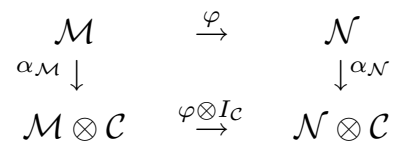

commutes. For further details on the theory of coalgebras, comodules and Hopf algebras we refer to the books of Abe [1] and Sweedler [25].

A very important construction for us is the cotensor product of comodules. Suppose that $\mathcal{M}$ and $\mathcal{N}$ are two comodules over the coalgebra $\mathcal{C}$ with coactions $\alpha_{\mathcal{M}}$ 
and $\alpha_{\mathcal{N}}$, respectively. Then the cotensor product of $\mathcal{M}$ and $\mathcal{N}$ is defined as the equalizer

$$
\mathcal{M} \otimes^{\mathcal{C}} \mathcal{N} \longrightarrow \mathcal{M} \otimes \mathcal{N} \underset{I_{\mathcal{M}} \otimes \alpha_{\mathcal{N}}^{\prime}}{\stackrel{\alpha_{\mathcal{M}} \otimes I_{\mathcal{N}}}{\longrightarrow}} \mathcal{M} \otimes \mathcal{C} \otimes \mathcal{N},
$$

i.e., $\mathcal{M} \otimes^{\mathcal{C}} \mathcal{N}=\left\{u \in \mathcal{M} \otimes \mathcal{N},\left(\alpha_{\mathcal{M}} \otimes I_{\mathcal{N}}\right) u=\left(I_{\mathcal{M}} \otimes \alpha_{\mathcal{N}}^{\prime}\right) u\right\}$. Here $\alpha_{\mathcal{N}}^{\prime}: \mathcal{N} \rightarrow$ $\mathcal{C} \otimes \mathcal{N}$ is the twisted comodule structure $\alpha_{\mathcal{N}}^{\prime}=\sigma_{12} \circ \alpha_{\mathcal{N}}$. Equivalently we have $\mathcal{M} \otimes{ }^{\mathcal{C}} \mathcal{N}=\operatorname{ker} \tau_{\mathcal{M} N}$, where $\tau_{\mathcal{M} N}=\alpha_{\mathcal{M}} \otimes I_{\mathcal{N}}-I_{\mathcal{M}} \otimes \alpha_{\mathcal{N}}^{\prime}$.

The following property of the cotensor product $\otimes^{\mathcal{C}}$ will be used later. It is a special case of Proposition 2.1.1 of [15].

Lemma 4.1. If $0 \rightarrow \mathcal{K} \rightarrow \mathcal{M} \stackrel{\varphi}{\rightarrow} \mathcal{N}$ is an exact sequence of comodules, i.e., $\mathcal{K}=$ $\operatorname{ker} \varphi$, and if $\mathcal{L}$ is another comodule, then $0 \rightarrow \mathcal{K} \otimes^{\mathcal{C}} \mathcal{L} \rightarrow \mathcal{M} \otimes^{\mathcal{C}} \mathcal{L} \rightarrow \mathcal{N} \otimes^{\mathcal{C}} \mathcal{L}$ is exact.

Proof. The diagram

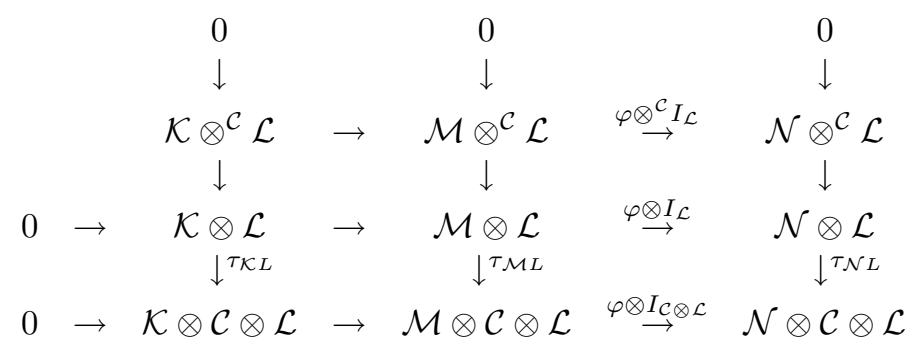

commutes and, by the definition of $\otimes^{\mathcal{C}}$, it has exact columns. Also the last two rows are exact, since $\mathcal{K}=\operatorname{ker} \varphi$. Then clearly $\mathcal{K} \otimes^{\mathcal{C}} \mathcal{L} \subset \operatorname{ker}\left(\varphi \otimes^{\mathcal{C}} I_{\mathcal{L}}\right)$, and an easy diagram chase shows that $\operatorname{ker}\left(\varphi \otimes^{\mathcal{C}} I_{\mathcal{L}}\right) \subset \mathcal{K} \otimes^{\mathcal{C}} \mathcal{L}$. Hence the assertion follows.

For a detailed discussion of the cotensor product $\otimes^{\mathcal{C}}$ we refer to [15]. See also $[21]$, where $\otimes^{\mathcal{C}}$ was introduced and is denoted by $\square_{\mathcal{C}}$.

\section{Root Subspaces as Comodules}

We turn our attention again to a multiparameter system (1). Suppose that $\mathcal{B}=\mathcal{B}_{\boldsymbol{\lambda}}$ is the coalgebra given by (15). Here we assume that $\boldsymbol{\lambda}$ is an eigenvalue of a multiparameter system. The tensor product $\mathcal{H}_{i}=H_{i} \otimes \mathcal{B}$ is then a cofree comodule $(i=1,2, \ldots, n)$ and so is $\mathcal{H}=H \otimes \mathcal{B}$.

A multiparameter system induces a system of linear transformations

$$
W_{i}(\mathbf{D})=V_{i 0}-\sum_{j=1}^{n} V_{i j} D_{j}
$$

$(i=1,2, \ldots, n)$, acting on the comodules $\mathcal{H}_{i}$. The operator $D_{j}$ is defined in (19). This system is extended in the obvious way to a system of linear transformations

$$
W_{i}(\mathbf{D})^{\dagger}=V_{i 0}^{\dagger}-\sum_{j=1}^{n} V_{i j}^{\dagger} D_{j}
$$

on $\mathcal{H}$, where $V_{i j}^{\dagger}$ are given by (2). Similarly the associated transformations $\Gamma_{i}$ of a multiparameter system induce linear transformations $\Gamma_{i}-D_{i}$ on $\mathcal{H}$. Here we use the same symbol for the induced linear transformation on a tensor product as for 
the original linear transformation when it is clear from the context which one is considered.

Here is our main result.

Theorem 5.1. Suppose that $\mathcal{R}_{i}=\operatorname{ker} W_{i}(\mathbf{D})(i=1,2, \ldots, n)$ and that

$$
\mathcal{R}_{\Gamma}=\bigcap_{i=1}^{n} \operatorname{ker}\left(\Gamma_{i}-D_{i}\right) .
$$

Then

$$
\mathcal{R}_{\Gamma}=\mathcal{R}_{1} \otimes^{\mathcal{B}} \otimes \mathcal{R}_{2} \otimes^{\mathcal{B}} \cdots \otimes^{\mathcal{B}} \mathcal{R}_{n}
$$

Proof. Note that the induced maps $V_{i j}$ on $\mathcal{H}_{i}$ and $V_{i j}^{\dagger}, \Gamma_{i}$ and $D_{i}$ on $\mathcal{H}$ are comodule homomorphisms. Hence also $W_{i}(\mathbf{D})$ and $\Gamma_{i}-D_{i}$ are comodule homomorphisms, and their kernels are subcomodules of $\mathcal{H}_{i}$ and $\mathcal{H}$, respectively. The commutativity of diagram (6) implies that the diagram

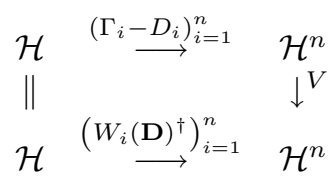

commutes. Because $V$ is invertible it follows that

$$
\mathcal{R}_{\Gamma}=\bigcap_{i=1}^{n} \mathcal{R}_{i}^{\dagger},
$$

where $\mathcal{R}_{i}^{\dagger}=\operatorname{ker} W_{i}(\mathbf{D})^{\dagger}$. Note that $\mathcal{R}_{1}^{\dagger}=\mathcal{R}_{1} \otimes^{\mathcal{B}} \mathcal{H}_{2} \otimes^{\mathcal{B}} \cdots \otimes^{\mathcal{B}} \mathcal{H}_{n}$ because the cotensor product preserves kernels by Lemma 4.1. Next we suppose that for $k<n$ we have that

$$
\bigcap_{i=1}^{k} \mathcal{R}_{i}^{\dagger}=\mathcal{R}_{1} \otimes^{\mathcal{B}} \cdots \otimes^{\mathcal{B}} \mathcal{R}_{k} \otimes^{\mathcal{B}} \mathcal{H}_{k+1} \otimes^{\mathcal{B}} \cdots \otimes^{\mathcal{B}} \mathcal{H}_{n} .
$$

We want to show that (26) holds for $k$ replaced by $k+1$ and thus prove the theorem by induction on $k$. Because the cotensor product preserves kernels (Lemma 4.1) it follows that the diagram

$$
\begin{aligned}
& 0 \quad 0 \quad 0 \\
& \downarrow \quad \downarrow \quad \downarrow \\
& \begin{array}{llllllll}
0 & \mathcal{R}_{k} \otimes{ }^{\mathcal{B}} \mathcal{R}_{k+1} & \rightarrow & \mathcal{H}_{k} \otimes \mathcal{B} & \mathcal{R}_{k+1} & \stackrel{W_{k}(\mathbf{D})^{\dagger}}{\rightarrow} & \mathcal{H}_{k} \otimes \mathcal{B} & \mathcal{R}_{k+1} \\
\downarrow & \downarrow & & & \downarrow
\end{array} \\
& 0 \quad \rightarrow \quad \mathcal{R}_{k} \otimes^{\mathcal{B}} \mathcal{H}_{k+1} \quad \rightarrow \quad \mathcal{H}_{k} \otimes{ }^{\mathcal{B}} \mathcal{H}_{k+1} \stackrel{W_{k}(\mathbf{D})^{\dagger}}{\longrightarrow} \quad \mathcal{H}_{k} \otimes^{\mathcal{B}} \mathcal{H}_{k+1} \\
& \downarrow^{W_{k+1}(\mathbf{D})^{\dagger}} \quad \downarrow^{W_{k+1}(\mathbf{D})^{\dagger}} \quad \downarrow^{W_{k+1}(\mathbf{D})^{\dagger}} \\
& 0 \quad \rightarrow \quad \mathcal{R}_{k} \otimes^{\mathcal{B}} \mathcal{H}_{k+1} \quad \rightarrow \quad \mathcal{H}_{k} \otimes^{\mathcal{B}} \mathcal{H}_{k+1} \stackrel{W_{k}(\mathbf{D})^{\dagger}}{\longrightarrow} \quad \mathcal{H}_{k} \otimes^{\mathcal{B}} \mathcal{H}_{k+1}
\end{aligned}
$$

commutes and has exact rows and columns. This remains valid upon cotensoring all the spaces in the above diagram by $\mathcal{R}_{1} \otimes^{\mathcal{B}} \cdots \otimes^{\mathcal{B}} \mathcal{R}_{k-1}$ on the left-hand side and by $\mathcal{H}_{k+2} \otimes^{\mathcal{B}} \cdots \otimes^{\mathcal{B}} \mathcal{H}_{n}$ on the right-hand side. Then it follows that $\bigcap_{i=1}^{k+1} \mathcal{R}_{i}^{\dagger}=$ $\mathcal{R}_{1} \otimes^{\mathcal{B}} \cdots \otimes^{\mathcal{B}} \mathcal{R}_{k+1} \otimes^{\mathcal{B}} \mathcal{H}_{k+2} \otimes^{\mathcal{B}} \cdots \otimes^{\mathcal{B}} \mathcal{H}_{n}$. When $k+1=n$ we have that $\bigcap_{i=1}^{n} \mathcal{R}_{i}^{\dagger}=$ $\mathcal{R}_{1} \otimes{ }^{\mathcal{B}} \mathcal{R}_{2} \otimes \mathcal{B} \cdots \otimes{ }^{\mathcal{B}} \mathcal{R}_{n}$, and so by $(25) \mathcal{R}_{\Gamma}=\mathcal{R}_{1} \otimes{ }^{\mathcal{B}} \mathcal{R}_{2} \otimes \otimes^{\mathcal{B}} \cdots \otimes \otimes^{\mathcal{B}} \mathcal{R}_{n}$. 
Next we prove an auxiliary result that connects the comodules $\mathcal{R}^{(l)}$ with the root subspaces $R_{l}$ defined by (8). A version of this result was proved in [13, Lemma]. Now the standard indexing on the filtration $\mathcal{R}^{(l)}$ is carried over to $R_{l}$ and thus the eigenspace $R_{0}$ becomes the 0 -th root subspace rather than the first root subspace.

Theorem 5.2. The linear map $\varepsilon^{\dagger}: \mathcal{R}^{(l)} \rightarrow R_{l}$, given by restricting $I_{H} \otimes \varepsilon: \mathcal{H} \rightarrow H$ to $\mathcal{R}^{(l)}$, is invertible.

We shall prove the theorem only for $n=2$. The general case is similar. Before we proceed we introduce some notation and prove an auxiliary result.

Suppose that $\boldsymbol{\lambda}=\left(\lambda_{1}, \lambda_{2}\right)$ is an eigenvalue of $(1)$. Then the coalgebra $\mathcal{B}^{(l)}=\mathcal{B}_{\boldsymbol{\lambda}}^{(l)}$, defined by (18), has a basis $\left\{\sum_{r+s=k} e_{r}^{1} \otimes e_{s}^{2}\right\}_{k=0}^{l}$, where $e_{m}^{i}=e_{m}\left(\lambda_{i}\right)$ for $i=1,2$ and $m \geq 0$. The following result describes the structure of the elements in $\mathcal{R}_{\Gamma}$.

Lemma 5.3. Suppose that $u=\sum_{r+s \leq l} u_{r s} \otimes e_{r}^{1} \otimes e_{s}^{2} \in \mathcal{R}_{\Gamma}$. Then

$$
\left(\Gamma_{1}-\lambda_{1} I\right) u_{r s}=u_{r+1, s} \quad \text { and } \quad\left(\Gamma_{2}-\lambda_{2} I\right) u_{r s}=u_{r, s+1},
$$

where $0 \leq r+s \leq l$, and $u_{r s}=0$ if $r+s>l$.

Proof. Recall that $\mathcal{R}_{\Gamma}=\bigcap_{i=1}^{2} \operatorname{ker}\left(\Gamma_{i}-D_{i} I\right)$. Then it follows that

$$
\begin{gathered}
0=\left(\Gamma_{i}-D_{i}\right) u=\sum_{r+s \leq l}\left[\Gamma_{1} u_{r s} \otimes e_{r}^{1} \otimes e_{s}^{2}-u_{r s} \otimes\left(\lambda_{1} e_{r}^{1}+e_{r-1}^{1}\right) \otimes e_{s}^{2}\right] \\
=\sum_{r+s \leq l}\left(\left(\Gamma_{1}-\lambda_{1} I\right) u_{r s}-u_{r+1, s}\right) \otimes e_{r}^{1} \otimes e_{s}^{2} .
\end{gathered}
$$

Therefore $\left(\Gamma_{1}-\lambda_{1} I\right) u_{r s}=u_{r+1, s}$, since $e_{r}^{1} \otimes e_{s}^{2}$ are linearly independent. The proof of relation (27) for $\Gamma_{2}$ is the same.

Proof of Theorem 5.2. Define $\eta: R_{l} \rightarrow \mathcal{R}^{(l)}$ by

$$
\eta(u)=\sum_{r+s \leq l}\left(\Gamma_{1}-\lambda_{1} I\right)^{r}\left(\Gamma_{2}-\lambda_{2}\right)^{s} u \otimes e_{r}^{1} \otimes e_{s}^{2} .
$$

Then it follows that $\left(\varepsilon^{\dagger} \circ \eta\right) u=u$, and by Lemma 5.3 also that

$$
\left(\eta \circ \varepsilon^{\dagger}\right)\left(\sum_{r+s \leq l} u_{r s} \otimes e_{r}^{1} \otimes e_{s}^{2}\right)=\sum_{r+s \leq l} u_{r s} \otimes e_{r}^{1} \otimes e_{s}^{2} .
$$

Thus $\eta$ is the inverse of $\varepsilon^{\dagger}$.

Consider the exact sequence

$$
0 \rightarrow \mathcal{R}^{\mathrm{o}} \rightarrow \mathcal{H}^{\mathrm{o}} \stackrel{\left(\Gamma_{i}-D_{i}\right)_{i}^{n}}{\longrightarrow}{ }^{n}\left(\mathcal{H}^{\mathrm{o}}\right)^{n},
$$

where $\mathcal{H}^{\mathrm{o}}=H \otimes F\left[x_{1}, x_{2}, \ldots, x_{n}\right]^{\mathrm{o}}$ is cofree and $\mathcal{R}^{\mathrm{o}}=\bigcap_{i=1}^{n} \operatorname{ker}\left(\Gamma_{i}-D_{i}\right)$. If $F$ is algebraically closed then (16) induces the direct sum decomposition

$$
\mathcal{R}^{\circ}=\bigoplus_{\boldsymbol{\lambda}} \mathcal{R}_{\boldsymbol{\lambda}}
$$

where $\mathcal{R}_{\boldsymbol{\lambda}}$ is defined as in (22), now indexed by $\boldsymbol{\lambda}$, and the sum is over all eigenvalues of the commuting system $\left\{\Gamma_{i}\right\}$. The following result is an immediate consequence of Theorems 5.1 and 5.2. Here, for each $i$,

$$
0 \rightarrow \mathcal{R}_{i \boldsymbol{\lambda}} \rightarrow H_{i} \otimes \mathcal{B}_{\boldsymbol{\lambda}} \stackrel{W_{i}(\mathbf{D})}{\longrightarrow} H_{i} \otimes \mathcal{B}_{\boldsymbol{\lambda}}
$$


is exact and $R_{\boldsymbol{\lambda}}=\varepsilon^{\dagger}\left(\mathcal{R}_{1 \boldsymbol{\lambda}} \otimes{ }^{\mathcal{B}_{\boldsymbol{\lambda}}} \mathcal{R}_{2 \boldsymbol{\lambda}} \otimes{ }^{\mathcal{B}} \boldsymbol{\lambda} \cdots \otimes{ }^{\mathcal{B}} \boldsymbol{\lambda} \mathcal{R}_{n \boldsymbol{\lambda}}\right)$.

Corollary 5.4. If $F$ is algebraically closed then the vector space $H$ has a direct sum decomposition

$$
H=\bigoplus_{\lambda} R_{\lambda}
$$

where the sum is over all eigenvalues $\boldsymbol{\lambda}$ of the system $\left\{W_{i}\right\}$.

Corollary 5.4 was proved already by Atkinson [5, Theorem 6.9.2]. Note that describing a basis for each $R_{\boldsymbol{\lambda}}$ (as we do in $\S 7$ ) then gives a completeness result for regular multiparameter systems.

If $F$ is not algebraically closed then

$$
F[x]^{\circ}=\bigoplus_{q} \mathcal{B}_{q}
$$

where the direct sum is over all the irreducible polynomials $q$ over $F$. Here $\mathcal{B}_{q}=$ $\bigcup_{l=0}^{\infty} \mathcal{B}_{q}^{(l)}$, where $\mathcal{B}_{q}^{(l)}$ is the vector space of all the linear functionals on $F[x] / \mathcal{I}$ and $\mathcal{I}$ is the ideal generated by $q^{l+1}$. (For details see [14].) As in (16), (29) induces a direct sum decomposition

$$
F\left[x_{1}, x_{2}, \ldots, x_{n}\right]^{\circ}=\bigoplus_{\mathbf{q}} \mathcal{B}_{\mathbf{q}}
$$

where $\mathbf{q}=\left(q_{1}, q_{2}, \ldots, q_{n}\right)$ runs over all $n$-tuples of irreducible polynomials (in one variable) over $F$ and $\mathcal{B}_{\mathbf{q}}=\mathcal{B}_{q_{1}} \otimes \mathcal{B}_{q_{2}} \otimes \cdots \otimes \mathcal{B}_{q_{n}}$. Theorem 5.1 and Corollary 5.4 remain valid in the case of a general field $F$ if $\boldsymbol{\lambda}$ is replaced by $\mathbf{q}$ in their statements. Here we only need to consider $\mathbf{q}=\left(q_{1}, q_{2}, \ldots, q_{n}\right)$ such that $\bigcap_{i=1}^{n} \operatorname{ker} q_{i}\left(\Gamma_{i}\right) \neq\{0\}$.

We also remark that Theorem 5.1 remains valid if the coalgebra $\mathcal{B}$ is replaced by another subcoalgebra of $F\left[x_{1}, x_{2}, \ldots, x_{n}\right]^{\circ}$. In particular, setting $\mathcal{B}=\mathcal{B}^{(0)}(\cong F)$ in (23) of Theorem 5.1, one gets (7), that is, Atkinson's Theorem [5, Th. 6.9.1]. Theorem 5.1 enables us to construct root subspaces associated with a multiparameter system without constructing the operators $\Gamma_{i}$. One only has to verify that the multiparameter system is regular in order to get the completeness result (28). The right-hand side of (23) is defined even when the multiparameter system is not regular, and one can still define root subspaces corresponding to any $\boldsymbol{\lambda} \in F^{n}$ (or q). However, we shall not study properties of root subspaces in such generality in this paper.

\section{The Structure of the Root Subspaces}

In this and the following sections we assume that $n=2$. For a general $n$ the ideas used in the presentation remain the same, but the constructions are of course technically more complex.

Assume that $\boldsymbol{\lambda}=\left(\lambda_{1}, \lambda_{2}\right)$ is an eigenvalue of (1) with ascent $L$. For $l=$ $0,1, \ldots, L$ and $i=1,2$ define $\mathcal{R}_{i}^{(l)}=\mathcal{R}_{i} \cap \mathcal{H}_{i}^{(l)}$ and $\mathcal{R}^{(l)}=\left(\mathcal{R}_{1} \otimes \mathcal{B} \mathcal{R}_{2}\right) \cap \mathcal{H}^{(l)}$, where the comodules $\mathcal{H}_{i}^{(l)}=H_{i} \otimes \mathcal{B}^{(l)}(i=1,2)$ and $\mathcal{H}^{(l)}=H \otimes \mathcal{B}^{(l)}$ are cofree. Note that $\mathcal{R}^{(L)}=\mathcal{R}=\mathcal{R}_{1} \otimes^{\mathcal{B}} \mathcal{R}_{2}$. Now we describe the structure of the elements of $\mathcal{R}_{i}^{(l)}$ and $\mathcal{R}^{(l)}$.

Lemma 6.1. A vector $v_{i}=\sum_{r+s \leq l} v_{r s}^{i} \otimes e_{r}^{1} \otimes e_{s}^{2}$ is in $\mathcal{R}_{i}^{(l)}$ if and only if

$$
W_{i}(\boldsymbol{\lambda}) v_{r s}^{i}+V_{i 1} v_{r+1, s}^{i}+V_{i 2} v_{r, s+1}^{i}=0 \text {, }
$$


for all $r, s$ such that $0 \leq r+s \leq l$. Here $v_{r s}=0$ if $r+s>l$.

Similarly, $u=\sum_{r+s \leq l} u_{r s} \otimes e_{r}^{1} \otimes e_{s}^{2}$ is in $\mathcal{R}^{(l)}$ if and only if

$$
W_{i}(\boldsymbol{\lambda})^{\dagger} u_{r s}+V_{i 1}^{\dagger} u_{r+1, s}+V_{i 2}^{\dagger} u_{r, s+1}=0,
$$

for $i=1,2$ and all $r, s$ such that $0 \leq r+s \leq l$. Here $u_{r s}=0$ if $r+s>l$.

Proof. Since $v_{i} \in \mathcal{R}_{i}=\operatorname{ker} W_{i}(\mathbf{D})$ it follows by (14) that

$$
\begin{aligned}
& 0=- W_{i}(\mathbf{D}) v_{i}=\sum_{r+s \leq l}\left[-V_{i 0} v_{r s}^{i} \otimes e_{1}^{r} \otimes e_{2}^{s}\right. \\
&\left.+V_{i 1} v_{r s}^{i} \otimes\left(\lambda_{1} e_{r}^{1}+e_{r-1}^{1}\right) \otimes e_{s}^{2}+V_{i 2} v_{r s}^{i} \otimes e_{r}^{1} \otimes\left(\lambda_{2} e_{s}^{2}+e_{s-1}^{2}\right)\right] \\
&=\sum_{r+s \leq l} W_{i}(\boldsymbol{\lambda}) v_{r s}^{i} \otimes e_{r}^{1} \otimes e_{s}^{2}+V_{i 1} v_{r+1, s}^{i} \otimes e_{r}^{1} \otimes e_{s}^{2}+V_{i 2} v_{r, s+1}^{i} \otimes e_{r}^{1} \otimes e_{s}^{2},
\end{aligned}
$$

and (31) for all $r, s$ is then equivalent to $v_{i} \in \mathcal{R}_{i}^{(l)}$ because of the linear independence of the $e_{r}^{1} \otimes e_{s}^{2}$. The proof of the second part of the lemma is similar.

From the definition of $\mathcal{R}$ it follows that $\Gamma_{i} u=D_{i} u$ for $u \in \mathcal{R}$. Lemma 5.3 implies then that $T_{i}=D_{i}-\lambda_{i} I=\Gamma_{i}-\lambda_{i} I$ maps $\mathcal{R}^{(l+1)}$ to $\mathcal{R}^{(l)}$ for $l \geq 0$, and that the diagram

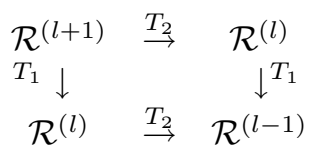

commutes for $l>0$.

The following result is an immediate consequence of Lemma 6.1.

Lemma 6.2. Suppose that $\pi^{(l)}: \mathcal{H}^{(l+1)} \rightarrow \mathcal{H}^{(l)}$ is the canonical projection given by $\pi^{(l)}\left(\sum_{r+s \leq l+1} u_{r s} \otimes e_{r}^{1} \otimes e_{s}^{2}\right)=\sum_{r+s \leq l} u_{r s} \otimes e_{r}^{1} \otimes e_{s}^{2}$. Then the diagram

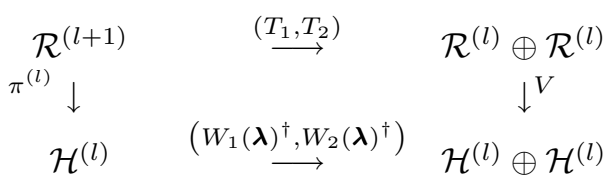

commutes.

Suppose that $T=\left(T_{1}, T_{2}\right): \mathcal{R}^{(l+1)} \rightarrow \mathcal{R}^{(l)} \oplus \mathcal{R}^{(l)}$ is defined by $T u=\left(T_{1} u, T_{2} u\right)$ and

$$
\mathcal{R}^{(l)} \oplus_{T} \mathcal{R}^{(l)}=\left\{\left(u_{1}, u_{2}\right) \in \mathcal{R}^{(l)} \oplus \mathcal{R}^{(l)} ; T_{2} u_{1}=T_{1} u_{2}\right\} .
$$

Then $\operatorname{im} T \subset \mathcal{R}^{(l)} \oplus_{T} \mathcal{R}^{(l)}$. Denote the kernel of the composite linear map

$$
\mathcal{R}^{(l)} \oplus_{T} \mathcal{R}^{(l)} \stackrel{V}{\rightarrow} \mathcal{H}^{(l)} \oplus \mathcal{H}^{(l)} \stackrel{\left(q_{1}, q_{2}\right)}{\longrightarrow}\left(\mathcal{H}^{(l)} / \operatorname{im} W_{1}(\boldsymbol{\lambda})^{\dagger}\right) \oplus\left(\mathcal{H}^{(l)} / \operatorname{im} W_{2}(\boldsymbol{\lambda})^{\dagger}\right)
$$

by $\mathcal{N}^{(l)}$. Here $q_{i}: \mathcal{H}^{(l)} \rightarrow \mathcal{H}^{(l)} / \operatorname{im} W_{i}(\boldsymbol{\lambda})^{\dagger}$ is the canonical projection.

Lemma 6.3. The kernel of $T: \mathcal{R}^{(l+1)} \rightarrow \mathcal{R}^{(l)} \oplus \mathcal{R}^{(l)}$ is equal to $\mathcal{R}^{(0)}$, and its image is $\mathcal{N}^{(l)}$. 
Proof. Note that

$$
T\left(\sum_{r+s \leq l+1} u_{r s} \otimes e_{r}^{1} \otimes e_{s}^{2}\right)=\left(\sum_{r+s \leq l} u_{r+1, s} \otimes e_{r}^{1} \otimes e_{s}^{2}, \sum_{r+s \leq l} u_{r, s+1} \otimes e_{r}^{1} \otimes e_{s}^{2}\right) .
$$

Then it follows that $\operatorname{ker} T=\mathcal{R}^{(0)}$. Lemma 6.2 implies that $\operatorname{im} T \subset \mathcal{N}^{(l)}$. To prove the converse relation assume that $\left(u_{1}, u_{2}\right) \in \mathcal{N}^{(l)}$. Then there exists $w=$ $\sum_{r+s \leq l} w_{r s} \otimes e_{r}^{1} \otimes e_{s}^{2} \in \mathcal{H}^{(l)}$ such that $V_{i 1}^{\dagger} u_{1}+V_{i 2}^{\dagger} u_{2}+W_{i}(\boldsymbol{\lambda})^{\dagger} w=0$, and in particular $V_{i 1}^{\dagger} u_{00}^{1}+V_{i 2}^{\dagger} u_{00}^{2}+W_{i}(\boldsymbol{\lambda})^{\dagger} w_{00}=0$. By Lemma 6.1 we have that

$$
\bar{u}=w_{00} \otimes e_{0}^{1} \otimes e_{0}^{2}+\sum_{r+s \leq l} u_{r s}^{1} \otimes e_{r+1}^{1} \otimes e_{s}^{2}+\sum_{k=0}^{l} u_{0 k}^{2} \otimes e_{0}^{1} \otimes e_{k+1}^{2}
$$

is in $\mathcal{R}^{(l+1)}$. Furthermore, $T \bar{u}=\left(u_{1}, u_{2}\right)$ and so $\mathcal{N}^{(l)} \subset \operatorname{im} T$.

Suppose that $\left(u_{1}, u_{2}\right) \in \mathcal{N}^{(l)}$ and that $w$ and $\bar{u}$ are as in the proof of the previous lemma. Then we define $S\left(u_{1}, u_{2}\right)=\bar{u}$. Note that the vector $w_{00}$ is not uniquely determined. So in order to define the map $S$ unambiguously we first define it on a basis for $\mathcal{N}^{(l)}$ and then extend it by linearity. Then we have :

Lemma 6.4. The composite map $T \circ S$ is the identity on $\mathcal{N}^{(l)}$, and $(S \circ T) u-u \in$ $\mathcal{R}^{(0)}$ for $u \in \mathcal{R}^{(l+1)}$.

The subspace $\mathcal{R}_{i}^{(l)} \oplus_{T} \mathcal{R}_{i}^{(l)}$ of $\mathcal{R}_{i}^{(l)} \oplus \mathcal{R}_{i}^{(l)}$ is defined by replacing $\mathcal{R}$ by $\mathcal{R}_{i}$ in (33). Then the identifications

$$
\left(\mathcal{R}_{1} \otimes^{\mathcal{B}}\left(\mathcal{R}_{2} \oplus \mathcal{R}_{2}\right)\right)^{(l)} \equiv \mathcal{R}^{(l)} \oplus \mathcal{R}^{(l)} \equiv\left(\left(\mathcal{R}_{1} \oplus \mathcal{R}_{1}\right) \otimes{ }^{\mathcal{B}} \mathcal{R}_{2}\right)^{(l)}
$$

induce identifications

$$
\left(\mathcal{R}_{1} \otimes^{\mathcal{B}}\left(\mathcal{R}_{2} \oplus_{T} \mathcal{R}_{2}\right)\right)^{(l)} \equiv \mathcal{R}^{(l)} \oplus_{T} \mathcal{R}^{(l)} \equiv\left(\left(\mathcal{R}_{1} \oplus_{T} \mathcal{R}_{1}\right) \otimes^{\mathcal{B}} \mathcal{R}_{2}\right)^{(l)}
$$

because $D_{i}$ acts on $H$ as identity. Suppose that the composite linear map

$$
\mathcal{R}_{i}^{(l)} \oplus_{T} \mathcal{R}_{i}^{(l)} \stackrel{V_{i}}{\rightarrow} \mathcal{H}_{i}^{(l)} \stackrel{q_{i}}{\rightarrow} \mathcal{H}_{i}^{(l)} / \mathrm{im} W_{i}(\boldsymbol{\lambda}),
$$

where $V_{i}$ is given by $V_{i}\left(v_{1}, v_{2}\right)=V_{i 1} v_{1}+V_{i 2} v_{2}$, has kernel $\mathcal{N}_{i}^{(l)}$. Then it follows via the identification (34) that

$$
\mathcal{N}^{(l)}=\left(\mathcal{R}_{1} \otimes^{\mathcal{B}} \mathcal{N}_{2}\right)^{(l)} \cap\left(\mathcal{N}_{1} \otimes^{\mathcal{B}} \mathcal{R}_{2}\right)^{(l)}\left(\subset \mathcal{R}^{(l)} \oplus_{T} \mathcal{R}^{(l)}\right)
$$

\section{Algorithm to Construct Bases for Root Subspaces}

In this section we present an algorithm for the construction of a basis for the root subspaces of multiparameter systems. From Theorem 5.2 it follows that the filtration on $\mathcal{R}$ carried over by $\varepsilon^{\dagger}$ to $H$ induces the filtration of the root subspaces given by (8). Thus if $\mathbf{B}^{(l)}$ is a basis for $\mathcal{R}^{(l)}(l=0,1, \ldots, L)$, then $\varepsilon^{\dagger}\left(\mathbf{B}^{(l)}\right)$ is a basis for $R_{l}$.

Algorithm 7.1. Step I. Find a basis $b_{i 0}=\left\{v_{i 0}^{k}\right\}_{k=1}^{j_{i}}$ for $\operatorname{ker} W_{i}(\boldsymbol{\lambda})(i=1,2)$. Here $j_{i}=\operatorname{dim} \operatorname{ker} W_{i}(\boldsymbol{\lambda})$. Then

$$
\mathbf{B}^{(0)}=\left\{u_{k l}=v_{10}^{k} \otimes v_{20}^{l} \otimes e_{0}^{1} \otimes e_{0}^{2}, k=1,2, \ldots, j_{1}, l=1,2, \ldots, j_{2}\right\}
$$

is a basis for $\mathcal{R}^{(0)}$. Set $l=0$ and $\mathcal{N}^{(-1)}=\{0\}$ and $\mathbf{b}^{(-1)}=\emptyset$. 
Step II. Consider $V_{i}: \mathcal{R}_{i}^{(l)} \oplus_{T} \mathcal{R}_{i}^{(l)} \rightarrow \mathcal{H}_{i}^{(l)} / \mathrm{im} W_{i}(\boldsymbol{\lambda})$ given by $V_{i}\left(v_{1}, v_{2}\right)=$ $V_{i 1} v_{1}+V_{i 2} v_{2}$. Denote the kernel of $V_{i}$ by $\mathcal{N}_{i}^{(l)}$ and write

$$
\mathcal{N}^{(l)}=\left(\mathcal{R}_{1} \otimes^{\mathcal{B}} \mathcal{N}_{2}\right)^{(l)} \cap\left(\mathcal{N}_{1} \otimes \otimes^{\mathcal{B}} \mathcal{R}_{2}\right)^{(l)}\left(\subset \mathcal{R}^{(l)} \oplus \mathcal{R}^{(l)}\right) .
$$

If $\mathcal{N}^{(l)}=\mathcal{N}^{(l-1)}$ then go to Step IV; else proceed with Step III.

Step III. Find a basis $\mathbf{b}_{i}^{(l)}$ for $\mathcal{N}_{i}^{(l)}$. Complete the basis $\mathbf{b}^{(l-1)}$ for $\mathcal{N}^{(l-1)}$ to a basis $\mathbf{b}^{(l)}$ for $\mathcal{N}^{(l)}$ using the vectors from $\mathbf{b}_{1}^{(l)}$ and $\mathbf{b}_{2}^{(l)}$. Lift the set $\mathbf{b}^{(l)} \backslash \mathbf{b}^{(l-1)}$ via $S$ to a set $\overline{\mathbf{b}}^{(l+1)}$. Then $\mathbf{B}^{(l+1)}=\mathbf{B} \cup \overline{\mathbf{b}}^{(l+1)}$. Set $l=l+1$ and repeat Step II.

Step IV. Write $L=l$ and find $B=\varepsilon^{\dagger}\left(\mathbf{B}^{(L)}\right)$. Quit the algorithm.

The set $B$ is a basis for the root subspace of the multiparameter system (1) at $\boldsymbol{\lambda}$, and $L$ is the ascent of $\boldsymbol{\lambda}$.

Proof. By (7) it follows that $\mathbf{B}^{(0)}$ is a basis for $\mathcal{R}^{(0)}$. We proceed to prove that $\mathbf{B}^{(l)}$ is a basis for $\mathcal{R}^{(l)}$ by induction on $l$. Suppose that $\mathbf{B}^{(l)}$ is a basis for $\mathcal{R}^{(l)}$. Since $\mathbf{b}^{(l)}$ is a basis for $\mathcal{N}^{(l)}$, then Lemma 6.4 implies that $\overline{\mathbf{b}}^{(l+1)}=S\left(\mathbf{b}^{(l)} \backslash \mathbf{b}^{(l-1)}\right)$ is a basis for a complement of $\mathcal{R}^{(l)}$ in $\mathcal{R}^{(l+1)}$. Hence $\mathbf{B}^{(l+1)}=\mathbf{B}^{(l)} \cup \overline{\mathbf{b}}^{(l+1)}$ is a basis for $\mathcal{R}^{(l+1)}$. Finally, by Theorem $5.2, B$ is a basis for $R_{L}$.

We remark that $\varepsilon^{\dagger}\left(\mathbf{B}_{l}\right)$ for $l \geq 0$ obtained in Algorithm 7.1 is a basis for $R_{l}$. In particular, $\varepsilon^{\dagger}\left(\mathbf{B}_{1}\right)$ is the same as the basis described in [8, Theorem 6.3]. If $\boldsymbol{\lambda}$ is nonderogatory [19], then Algorithm 7.1 reduces to a procedure similar to [19, Algorithm 16].

For comparison with $[6,19]$ we consider [6, Example 4.4], which is [19, Example 20]. Here $F=\mathbb{C}$.

Example 7.2. The two-parameter system

$$
W_{1}(\boldsymbol{\lambda})=\left[\begin{array}{ll}
2 & 1 \\
1 & 2
\end{array}\right] \lambda_{1}+\left[\begin{array}{cc}
-1 & 0 \\
0 & -1
\end{array}\right] \lambda_{2}-\left[\begin{array}{ll}
1 & 0 \\
0 & 0
\end{array}\right]
$$

and

$$
W_{2}(\boldsymbol{\lambda})=\left[\begin{array}{cc}
-4 & 1 \\
1 & -4
\end{array}\right] \lambda_{1}+\left[\begin{array}{ll}
2 & 0 \\
0 & 2
\end{array}\right] \lambda_{2}-\left[\begin{array}{ll}
1 & 0 \\
0 & 0
\end{array}\right]
$$

has $\mathbf{0}$ as an eigenvalue. As in $[6,19]$ we choose $v_{0}^{i}=\left[\begin{array}{l}0 \\ 1\end{array}\right](i=1,2)$, which spans $\operatorname{ker} V_{i 0}$. We write $v_{i}=v_{0}^{i} \otimes e_{0} \otimes e_{0}$ and $y_{0}=v_{0}^{1} \otimes v_{0}^{2}$. Then $\mathbf{B}^{(0)}=\left\{u_{0}=y_{0} \otimes e_{0} \otimes e_{0}\right\}$. The subspace $\mathcal{R}_{i}^{(0)} \oplus_{d} \mathcal{R}_{i}^{(0)}$ is spanned by $\left(v_{i}, v_{i}\right)$, im $V_{i 0}=\left\{\left[\begin{array}{c}\alpha \\ 0\end{array}\right] \otimes e_{0} \otimes e_{0} ; \alpha \in \mathbb{C}\right\}$ and

$$
\mathcal{N}_{i}^{(0)}=\left\{\left(\left[\begin{array}{l}
0 \\
\frac{\alpha}{2}
\end{array}\right] \otimes e_{0} \otimes e_{0},\left[\begin{array}{l}
0 \\
\alpha
\end{array}\right] \otimes e_{0} \otimes e_{0}\right) ; \alpha \in \mathbb{C}\right\} .
$$

Then $\left(\frac{1}{2} u_{0}, u_{0}\right)$ is a basis for $\mathcal{N}^{(0)}$. It remains to lift $\left(\frac{1}{2} u_{0}, u_{0}\right)$ via $S$. To do so we choose $w_{i}=\left[\begin{array}{c}1 / 2 \\ 0\end{array}\right]$. Then

$$
u_{1}=S\left(\frac{1}{2} u_{0}, u_{0}\right)=y_{1} \otimes e_{0} \otimes e_{0}+y_{0} \otimes\left(\frac{1}{2} e_{1} \otimes e_{0}+\otimes e_{0} \otimes e_{1}\right)
$$

where $u_{1}=w_{1} \otimes v_{0}^{2}+v_{0}^{1} \otimes w_{2}$. Then $\mathbf{B}^{(1)}=\left\{u_{0}, u_{1}\right\}$. Next we find that $\mathcal{N}_{i}^{(1)}=\mathcal{N}_{i}^{(0)}$, and thus $L=1$ and $B=\varepsilon^{\dagger}\left(\mathbf{B}^{(1)}\right)=\left\{y_{0}, y_{1}\right\}$. We quit the algorithm. We remark that the basis $B$ is the same as that obtained in [6] and [19]. 


\section{Root Subspaces for Eigenvalues of Fredholm Type}

In this section we generalize our main result, Theorem 5.1, to a general Hilbert space setting. We assume that for $i=1,2, \ldots, n$ the linear maps $V_{i j}(j=$ $0,1, \ldots, n-1)$ are bounded on the Hilbert space $H_{i}$ and that the $V_{i n}$ are (possibly) unbounded, but closed with dense domain $C_{i} \subset H_{i}$.

Here our assumptions are as in [8]. Often in the literature the roles of $V_{i 0}$ and $V_{i n}$ are reversed. For us this is not important, since the discussion for the reversed case remains the same. We also remark that typically in the applications the $V_{i n}$ are differential operators and the $V_{i j}$ are multiplication operators.

The operators $V_{i j}(j=0,1, \ldots, n-1)$ induce operators $V_{i j}^{\dagger}$ acting on the Hilbert space $H=H_{1} \otimes H_{2} \otimes \cdots \otimes H_{n}$ as in (2), and similarly $V_{i n}$ induces an operator $V_{i n}^{\dagger}$ with domain $C_{i}^{\dagger} \subset H$. Because the (algebraic) tensor product $C_{1} \otimes C_{2} \otimes \cdots \otimes C_{n}$, which is dense in $H$, is a subspace of $C=\bigcap_{i=1}^{n} C_{i}^{\dagger}$, it follows that also $C$ is dense in $H$. Define the operators $\Delta_{i}(i=0,1, \ldots, n)$, now acting on $C$, as in $\S 2$. Further assume that $\Delta_{0}$ has bounded inverse, and let $\Gamma_{i}=\Delta_{0}^{-1} \Delta_{i}: C \rightarrow C$. The linear map $V$ defined as in (4) is now acting on $C^{n}$. Similarly we define a linear map $V_{0}: C^{n+1} \rightarrow H^{n}$ by the array

$$
V_{0}=\left[\begin{array}{cccc}
V_{10}^{\dagger} & V_{11}^{\dagger} & \cdots & V_{1 n}^{\dagger} \\
V_{20}^{\dagger} & V_{21}^{\dagger} & \cdots & V_{2 n}^{\dagger} \\
\vdots & \vdots & & \vdots \\
V_{n 0}^{\dagger} & V_{n 1}^{\dagger} & \cdots & V_{n n}^{\dagger}
\end{array}\right] .
$$

Suppose $K=P_{n+1}\left(\operatorname{ker} V_{0}\right) \subset C$, where $P_{n+1}$ is the projection of $C^{n+1}$ onto the last component. Then Theorem 3.2 of [8] states that $\Gamma_{i} \Gamma_{j} x=\Gamma_{j} \Gamma_{i} x$ for $x \in K$ and that the diagram

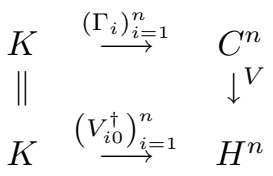

commutes. If $K$ is replaced by $C$ then the above diagram may not commute anymore. This is different from the finite-dimensional case (see (6)). At this stage additional regularity assumptions are necessary. We assume that $K=C$, but we comment on a more general case later. The assumption $K=C$ is satisfied in applications to boundary value problems (see e.g. $[8,10]$ ). We also remark that to study the commutativity of (36) under various assumptions is an important topic of multiparameter spectral theory (see [2, 18, 23]).

An eigenvalue $\boldsymbol{\lambda}$ of a multiparameter system (1) where $V_{i j}$ are as above is called semi-Fredholm if $W_{i}(\boldsymbol{\lambda})$ has finite-dimensional kernel for all $i$. Define the root subspaces $R_{l}$ corresponding to $\boldsymbol{\lambda}$ as in (8).

Lemma 8.1. Suppose that $\boldsymbol{\lambda}$ is semi-Fredholm. Then the root subspace $R_{l}(l \geq 0)$ is finite-dimensional.

Proof. Because $R_{l} \subset C=K$ it follows by Corollary 4.2 of [8] that

$$
R_{0}=\operatorname{ker} W_{1}(\boldsymbol{\lambda}) \otimes \operatorname{ker} W_{2}(\boldsymbol{\lambda}) \otimes \cdots \otimes \operatorname{ker} W_{n}(\boldsymbol{\lambda}) .
$$

Since $\boldsymbol{\lambda}$ is semi-Fredholm, $\operatorname{dim} R_{0}=\prod_{i=1}^{n} \operatorname{dim} \operatorname{ker} W_{i}(\boldsymbol{\lambda})$ is finite. We proceed by induction on $l$ using a similar argument as is used in [8] to prove Lemma 5.2 (which 
is our lemma for $l=1$ ). Suppose that $R_{l-1}$ is finite-dimensional. The range of $\left.\left(\Gamma_{j}-\lambda_{j}\right)\right|_{R_{l}}$ is a subspace of $R_{l-1}$ and so finite-dimensional. Then the orthogonal complement $Q_{j}$ of $\left.\operatorname{ker}\left(\Gamma_{j}-\lambda_{j}\right)\right|_{R_{l}}$ is finite-dimensional, and because $R_{l}=R_{0} \oplus Q$, where $Q=\sum_{j=1}^{n} Q_{j}$, also $R_{l}$ is finite-dimensional.

For $l \geq 0$ let $\mathcal{H}^{(m)}=H \otimes \mathcal{B}_{\lambda}^{(m)}$, where $\mathcal{B}_{\boldsymbol{\lambda}}^{(m)}$ is defined in (18). Similarly we define $\mathcal{C}_{i}^{(m)}, \mathcal{C}^{(m)}$ and $K^{(m)}$. The linear transformation $\Gamma_{i}-D_{i}$ is now acting on $\mathcal{C}^{(m)}$ while the linear transformations $W_{i}(\mathbf{D})$ and $W_{i}(\mathbf{D})^{\dagger}$, defined as in (20) and (21), are acting on $\mathcal{C}_{i}^{(m)}$ and $\mathcal{C}^{(m)}$, respectively. Then $\mathcal{R}_{i}^{(m)}=\operatorname{ker} W_{i}(\mathbf{D}) \subset$ $\mathcal{C}_{i}^{(m)}(i=1,2, \ldots, n)$ and $\mathcal{R}_{\Gamma}^{(m)}=\bigcap_{i=1}^{n} \operatorname{ker}\left(\Gamma_{i}-D_{i}\right) \subset \mathcal{C}^{(m)}$.

Theorem 8.2. Suppose that $\boldsymbol{\lambda}$ is a semi-Fredholm eigenvalue of a multiparameter system $W_{i}(\boldsymbol{\lambda})(i=1,2, \ldots, n)$. Then for each $l \geq 0$

$$
\mathcal{R}_{\Gamma}^{(l)}=\mathcal{R}_{1}^{(l)} \otimes^{\mathcal{B}^{(l)}} \mathcal{R}_{2}^{(l)} \otimes^{\mathcal{B}^{(l)}} \cdots \otimes^{\mathcal{B}^{(l)}} \mathcal{R}_{n}^{(l)} .
$$

Proof. Because diagram (36) commutes it follows that the diagram

$$
\begin{aligned}
& \begin{array}{ccc}
\mathcal{K}^{(l)} & \stackrel{\left(\Gamma_{i}-D_{i}\right)_{i=1}^{n}}{\longrightarrow} & \left(\mathcal{C}^{(l)}\right)^{n} \\
\| & \downarrow^{V}
\end{array} \\
& \mathcal{K}^{(l)} \stackrel{\left(W_{i}(\mathbf{D})^{\dagger}\right.}{\longrightarrow}{ }_{i=1}^{n} \quad\left(\mathcal{H}^{(l)}\right)^{n}
\end{aligned}
$$

also commutes. Since $V$ is one-to-one ( $\Delta_{0}$ has an inverse) and $R_{m} \subset C=K$, we see that $\mathcal{R}_{\Gamma}^{(l)}=\bigcap_{i=1}^{n} \mathcal{R}_{i}^{\dagger(l)}$, where $\mathcal{R}_{i}^{\dagger(l)}=\operatorname{ker} W_{i}(\mathbf{D})^{\dagger}$. To complete the proof, proceed by induction as in the proof of Theorem 5.1.

For relation (37) to hold it suffices to assume that $R_{l} \subset K$ instead of $K=C$. However, if $R_{l} \not \subset K$ then (37) does not hold in general any more, as Examples 4.4 and 5.1 of [8] show. Then the right-hand side of (37) is equal to $\mathcal{R}_{\Gamma}^{(l)} \cap \mathcal{K}^{(l)}$. We also remark that Algorithm 7.1 can be generalized directly to the setting considered in this section.

We conclude with some remarks. It has been already noticed in the literature $[6,11,16]$ that in order to find root vectors for the $\Gamma$ 's one has to consider the recursive systems of equations similar to (31) and (32) for $n=2$, and solve them for the vectors $v_{r s}^{i}$ and $u_{r s}$, respectively. However, except in some special cases mentioned later, no general method was known to construct root vectors for the $\Gamma$ 's given the vectors $v_{r s}^{i}$ or $u_{r s}$. It is shown in this paper how to find root vectors using the cotensor product of certain comodules associated with $W_{i}(\boldsymbol{\lambda})$. By Algorithm 7.1 one then finds bases for the root subspaces of the $\Gamma$ 's using the original operators $V_{i j}$, and that amounts to first solving the systems of equations (31) for $v_{r s}^{i}$, and then 'equalizing' these solutions for different $i$. In general, this is a technically involved process. Now it would be interesting to find classes of eigenvalues for which the structure of the root subspaces is simpler. Beside the semi-simple case, i.e., when the eigenvectors span the root subspaces (this happens for instance in the so-called right definite case $[5,26]$ ), there are two classes known in the literature. These are the class of real eigenvalues for the uniformly elliptic multiparameter systems, where Binding's construction provides bases for root subspaces [6, Theorem 3.4], and the class of nonderogatory eigenvalues, for which bases for root subspaces in finite-dimensions are constructed in [19, Algorithm 16]. 


\section{REFERENCES}

[1] E. Abe. Hopf Algebras. Cambridge Univ. Press, 1980. MR 83a:16010

[2] M.S. Almamedov and G.A. Isaev. Solvability of Nonselfadjoint Linear Operator Systems, and the Set of Decomposability of Multiparameter Spectral Problems. Soviet. Math. Dokl., 31:472-474, 1985. MR 87b:47018 (Russian original)

[3] F.M. Arscott. Periodic Differential Equations. Pergamon Press : Oxford, 1964. MR 30:4066

[4] F.V. Atkinson. Multiparameter Spectral Theory. Bull. Amer. Math. Soc., 74:1-27, 1968. MR 36:3145

[5] F.V. Atkinson. Multiparameter Eigenvalue Problems. Academic Press, 1972. MR 56:9291

[6] P.A. Binding. Multiparameter Root Vectors. Proc. Edin. Math. Soc., 32:19-29, 1989. MR 90a: 47048

[7] P.A. Binding and P.J. Browne. Two Parameter Eigenvalue Problems for Matrices. Lin. Alg. Appl., 113:139-157, 1989. MR 89m:47012

[8] P.A. Binding and T. Košir. Second Root Vectors for Multiparameter Eigenvalue Problems of Fredholm Type, Trans. Amer. Math. Soc. 348: 229-249, 1996.

[9] P.A. Binding and K. Seddighi. Elliptic Multiparameter Eigenvalue Problems. Proc. Edin. Math. Soc., 30:215-228, 1987. MR 88g:47036

[10] M. Faierman. Two-parameter Eigenvalue Problems in Ordinary Differential Equations, volume 205 of Pitman Research Notes in Mathematics. Longman Scientific and Technical, U.K., 1991. MR 93b:47095

[11] G.A. Gadzhiev. On a Multitime Equation and its Reduction to a Multiparameter Spectral Problem. Soviet. Math. Dokl., 32:710-713, 1985. MR 87j:35365 (Russian original)

[12] G.A. Gadzhiev. Introduction to Multiparameter Spectral Theory. Azerbaijan State University, Baku, 1987. (Russian)

[13] L. Grunenfelder and T. Košir. Representation of Commuting Maps by Tensor Products, preprint.

[14] L. Grunenfelder and M. Omladič. Linearly Recursive Sequences and Operator Polynomials. Lin. Alg. Appl., 182:127-145, 1993. MR 93m:16027

[15] L. Grunenfelder and R. Paré. Families Parametrized by Coalgebras. J. Algebra, 107:316-375, 1987. MR 88i:16044

[16] G.A. Isaev. On Root Elements of Multiparameter Spectral Problems. Soviet. Math. Dokl., 21:127-130, 1980. MR 82c:47021 (Russian original)

[17] H.(G.A.) Isaev. Lectures on Multiparameter Spectral Theory. Dept. of Math. and Stats., University of Calgary, 1985.

[18] A. Källström and B.D. Sleeman. Solvability of a Linear Operator System. J. Math. Anal. Appl., 55:785-793, 1976. MR 54:5881

[19] T. Košir. The Finite-dimensional Multiparameter Spectral Theory : The Nonderogatory Case. Lin. Alg. Appl., 212/213:45-70, 1994. MR 95k:15020

[20] T. Košir. Commuting Matrices and Multiparameter Eigenvalue Problems. PhD thesis, Dept. of Math. and Stats., University of Calgary, 1993.

[21] J.W. Milnor and J.C. Moore. On the Structure of Hopf Algebras. Ann. of Math., 81:211-264, 1965. MR 30:4259

[22] B. Peterson and E. Taft. The Hopf Algebra of Linearly Recursive Sequences. Aequationes Math., 20:1-17, 1980. MR 81j:16012

[23] B.D. Sleeman. Multiparameter Spectral Theory in Hilbert Space, volume 22 of Pitman Research Notes in Mathematics. Pitman, London, Belmont, 1978. MR 81h:47004

[24] B.D. Sleeman. Review of [10]. SIAM Review, 34:684-687, 1992.

[25] M.E. Sweedler. Hopf Algebras. Benjamin, New York, 1969. MR 40:5705

[26] H. Volkmer. On the Completeness of Eigenvectors of Right Definite Multiparameter Problems. Proc. Roy. Soc. Edin., 96A:69-78, 1984. MR 86a:47014

[27] H. Volkmer. Multiparameter Eigenvalue Problems and Expansion Theorems, volume 1356 of Lecture Notes in Mathematics. Springer-Verlag, Berlin, New York, 1988. MR 91d:47021

Department of Mathematics, Statistics and Computing Science, Dalhousie UniverSity, Halifax, Nova Scotia, Canada B3H 3J5

E-mail address: luzius@cs.dal.ca

Department of Mathematics, University of Ljubljana, Jadranska 19, 61000 LjublJANA, Sloevenia

E-mail address: tkosir@fmf.uni-lj.si 